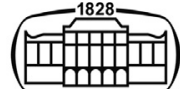

AKADÉMIAI KIADÓ

Journal of Behavioral Addictions

10 (2021) 4, 1005-1014

DOl:

$10.1556 / 2006.2021 .00071$

(C) 2021 The Author(s)

\section{FULL-LENGTH REPORT}

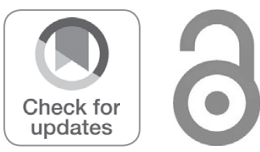

\title{
Efficacy of short-term telemedicine motivation- based intervention for individuals with Internet Use Disorder - A pilot-study
}

\author{
LAURA BOTTEL $^{1 *} \odot$, MATTHIAS BRAND ${ }^{3,4} \odot$, \\ JAN DIERIS-HIRCHE ${ }^{1} \odot$, STEPHAN HERPERTZ ${ }^{1} \odot$, \\ NINA TIMMESFELD ${ }^{5} \odot$ and BERT THEODOR TE WILDT ${ }^{1,2}$
}

\footnotetext{
${ }^{1}$ Ruhr University Bochum, LWL-University Hospital, Department of Psychosomatic Medicine and Psychotherapy, Bochum, Germany

${ }^{2}$ Psychosomatic Hospital Diessen Monastery, Diessen am Ammersee, Germany

${ }^{3}$ General Psychology: Cognition and Center for Behavioral Addiction Research (CeBAR), University of Duisburg-Essen, Duisburg, Germany

${ }^{4}$ Erwin L. Hahn Institute for Magnetic Resonance Imaging, Essen, Germany

${ }^{5}$ Ruhr University Bochum, Department of Medical Informatics, Biometry and Epidemiology, Bochum, Germany
}

Received: July 2, 2021 - Revised manuscript received: September 24, 2021 - Accepted: October 4, 2021 Published online: November 17, 2021

\begin{abstract}
Background: Internet Use Disorders (IUD) cover a range of online-related behavioral addictions, which are acknowledged and strengthened by the inclusion of (online) gaming disorder in the ICD-11 by the World Health Organization. Internet-based telemedicine interventions offer the possibility to reach out for individuals with IUD where the disorder emerges, in order to enhance their motivation to change their Internet use behavior. Methods: In the course of the short-term telemedicine motivation-based intervention, adult participants took part in two webcam-based counselling sessions based on Motivational Interviewing techniques. Two weeks after the first webcam-based counselling session the second took place. Participants completed questionnaires regarding the motivation to change their Internet use behavior (iSOCRATES) and symptoms of IUD (s-IAT) at three times of measurement: t0 (pre-intervention), t1 (mid-intervention) and $\mathrm{t} 2$ (post-intervention). Results: 73 affected individuals (83.6\% male, average age 35 years $(S D=12.49)$ took part in the whole intervention including the questionnaire-based post intervention survey ( $\mathrm{t} 2$ ). Over the course of the telemedicine intervention, a significant increase in the motivation to change with regard to the own Internet use behavior as well as a significant reduction in the symptom severity of an IUD and duration of Internet use (reduction of $2 \mathrm{hrs} / \mathrm{d}$ ) were shown. Conclusion: The telemedicine pilot study shows that online-based consultation can be effective and helpful for individuals with IUD. Therefore, such a telemedicine intervention may be a suitable extension to the already existing analogous care system.
\end{abstract}

\section{KEYWORDS}

telemedicine, eHealth, Internet addiction, Internet Use Disorder, gaming disorder, motivational interviewing

\section{INTRODUCTION}

Corresponding author. E-mail: laura.bottel@rub.de

In 2013, the American Psychiatric Association included Internet gaming disorder (IGD) in section III under "condition for further research" of the DSM-5 (American Psychatric Association, 2013) and in 2018 the World Health Organization decided the inclusion of (online) gaming disorder as formal disorder in the upcoming ICD-11 (World Health Organization, 2018) in the section "disorders due to addictive behaviours". Besides IGD, there are further 
specific kinds of Internet applications which can result in addictive behaviors like online shopping, online-pornography use, social media use as well as the unspecific, generalized use of the Internet (Brand et al., 2020; Montag et al., 2015; Potenza, Higuchi, \& Brand, 2018). Since various online applications can be used in a pathological way, the term Internet Use Disorder (IUD) is used frequently as an umbrella term for a pathological use of different Internet applications (Brand, Young, Laier, Wölfling, \& Potenza, 2016; Montag, Wegmann, Sariyska, Demetrovics, \& Brand, 2021).

The prevalence of IGD internationally ranges between $0.7 \%$ and $27.5 \%$ (Mihara \& Higuchi, 2017). Higher prevalence rates are seen for men than women and for younger compared to older individuals (Mihara \& Higuchi, 2017). Rumpf et al. (2014) estimated a prevalence of $1 \%$ for IUD and $4.6 \%$ for hazardous Internet use with no gender difference. Affected women primarily use social networking sites and the majority of men online-games (Rumpf et al., 2014). A meta-analysis on the effectiveness of cognitive behavioral therapy (CBT) for IGD summarized 12 studies and showed high effect sizes in reducing symptom severity of IGD (Stevens, King, Dorstyn, \& Delfabbro, 2019).

\section{Telemedicine and Motivational Interviewing for individuals with IUD}

Telemedicine interventions for individuals with IUD are rare and the quality standards of different telemedicine applications vary. The few existing telemedicine offerings for individuals with IUD are often defined by asynchronous communication via apps or support via e-mail (Chebli, Blaszczynski, \& Gainsbury, 2016; Lam \& Lam, 2016). The effectiveness of Motivational Interviewing (MI) techniques in the treatment and counselling of individuals with addictive behaviours (Bischof, G., Bischof, A., \& Rumpf, 2021; Diclemente, Corno, Graydon, Wiprovnick, \& Knoblach, 2017) and in particular IUD was verified (e.g. van Rooij, Zinn, Schoenmakers, \& van de Mheen, 2012). Recent studies demonstrated that MI techniques can not only be used effectively in direct face-to-face contact, but also in the course of telephone or Internet-based consultations (Bommelé et al., 2017; Jiang, Wu, \& Gao, 2017).

\section{Paradoxical or obvious? Reaching out to individuals with IUD online}

Counselling individuals with IUD online sounds paradoxical at first, however there are many aspects that support such an innovative approach and provide the basis for the telemedicine pilot study: (1) Specialized treatment and counselling services on-site for individuals with IUD has increased over the last few years, but there is not yet a comprehensive treatment structure (Saunders et al., 2017). (2) Similar to the "streetworking"-approach (Hill \& Laredo, 2019; Szeintuch, 2015), individuals with IUD spend a lot of time online, which makes it easier to reach out and get in touch for the first time. (3) Comorbidities such as anxiety disorders, depression or attention deficit hyperactivity disorder (ADHD) play a major role among affected individuals (Carli et al., 2013; Yen et al., 2008), which make it even more difficult for individuals with IUD to find their way into a conventional treatment setting. (4) Addictive disorders are often associated with strong feelings of guilt and shame, as affected individuals often experience failure (O’Connor, Berry, Inaba, Weiss, \& Morrison, 1994), which may make it even more difficult for them to consult a therapist offline or to exchange feelings and thoughts with relatives and friends. (5) Telemedicine services in treatment of depression and anxiety (Johansson \& Andersson, 2012; Van't Hof, Cuijpers, \& Stein, 2009) as well as in counselling and treatment of substance-use disorders (Kruse et al., 2020; Tait, Spijkerman, \& Riper, 2013) have already been shown to be effective. Based on these aspects, a low-threshold and easily accessible telemedicine health care solution for individuals with IUD has been developed.

\section{Study aim}

The aim of the telemedicine pilot study was to provide a low-threshold first point of contact for individuals with IUD who are ambivalent about changing their Internet use behavior. Therefor the primary aim of OASIS was to explore, if the developed telemedicine intervention with two webcam-based sessions may increase the motivation to change the dysfunctional Internet use behavior. The secondary aim was to find out whether the short-term telemedicine intervention (two webcam-based sessions) already reduces the symptom severity of IUD and decreases Internet usage times.

\section{METHODS}

\section{Procedure}

The telemedicine pilot study "Online-Ambulatory Service for Individuals with Internet Use Disorder" (OASIS) was conducted from September 2016 to December 2019. Consultants were psychologists, psychiatrists and experts in the field of counselling individuals with IUD. To ensure a high quality of the consultations, all consultants beforehand participated in MI techniques training, and regular supervision by the clinical project management (senior physician of the outpatient clinic and expert in the field of treatment individuals with IUD) took place during the recruitment phase.

Recruitment for study participation took place via the program's homepage. Extensive public relations, use of social networking sites, participation at the largest computer game convention, and project presentations to health care professionals raised awareness for the telemedicine pilot study. Interested persons were asked to fill out a screening questionnaire (nine DSM-5 diagnostic criteria of IGD formulated as questions for IUD answered on a 5-point Likert scale), create a user-account by providing their name, e-mail address and a pseudonym of their choice. After the participants provided informed consent and confirmed that they met the inclusion criteria, they could book an 
appointment for their first telemedicine consultation on the study-portal.

Before the first telemedicine consultation ( $t 0$ - preintervention), after the first ( $\mathrm{t} 1$ - mid-intervention) and second consultation ( $\mathrm{t} 2$ - post-intervention) and three months after the second consultation ( $\mathrm{t} 3$ - follow-up) participants were asked to answer several questions regarding their Internet use behaviors via the study-portal (see Fig. 1).

The telemedicine consultations (60 min each) took place via webcam using telemedicine software La-Well Systems $\mathrm{GmbH}$. A detailed data protection concept for handling and protecting the participants' data was developed and verified by the Competence Centre of Healthcare Telematics. The key elements of the study were two webcam-based online consultation sessions with a specialized consultant. In the first telemedicine consultation, a detailed media, social and family history was conducted. Based on structured interviews (M.I.N.I.) psychological comorbidities and IUD (American Psychatric Association, 2013) were assessed and it was ensured that the exclusion criteria for study participation (see next section) were not present. In the second telemedicine consultation (approximately 2 weeks after the first online consultation) individual treatment and support options on-site were presented and discussed (see Table 1).

\section{Participants}

All persons older than 18 years having access to the Internet, webcam and an e-mail account were principally able to participate in the study. Exclusion criteria were current psychotic or affective disorders, as well as primary substance-related disorders and/or suicidal ideations.

Since some participants did not take part in the complete intervention, two groups of participants were defined

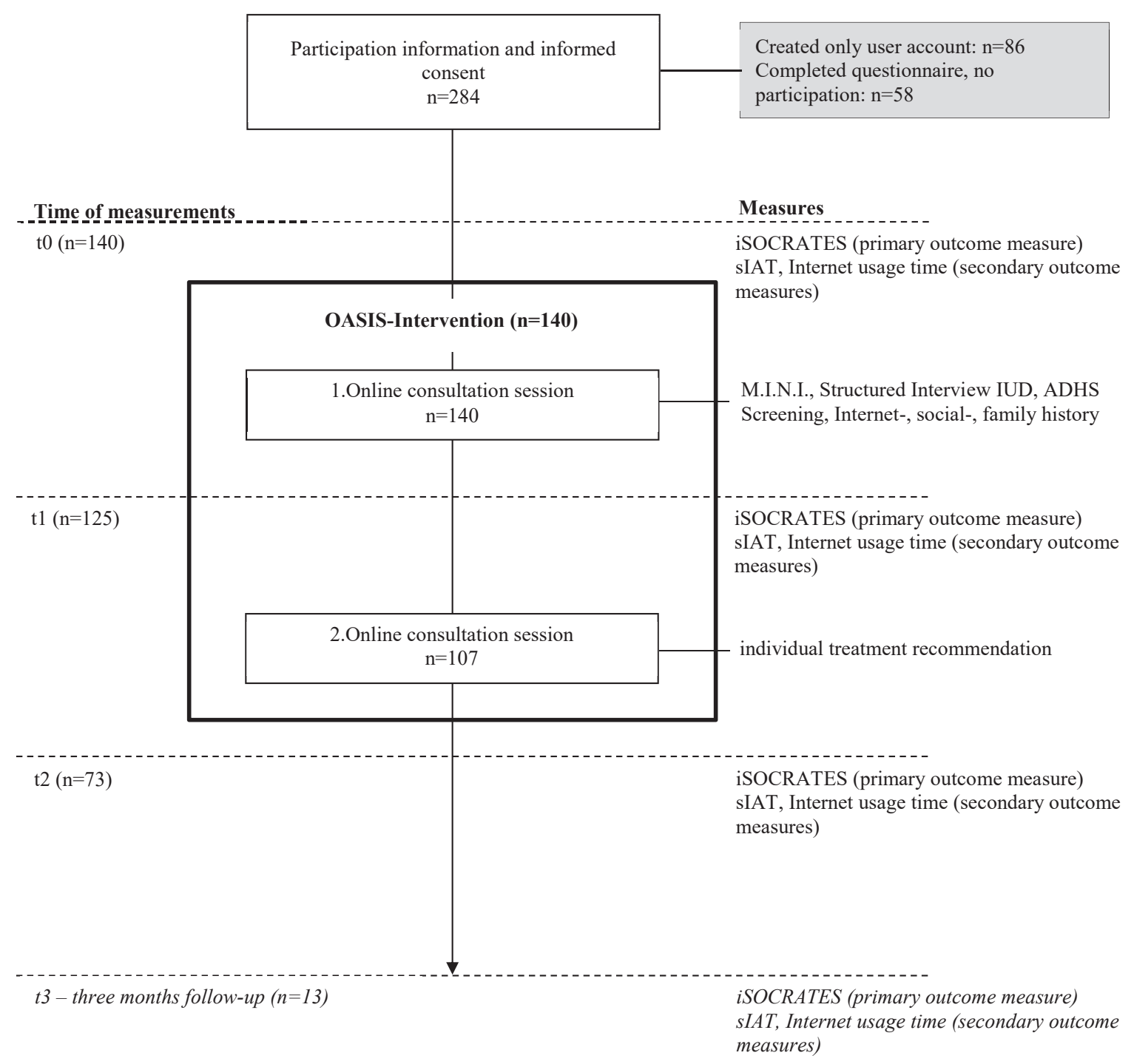

Fig. 1. Study design including times of measurement ( $\mathrm{t} 0$ - pre-intervention, $\mathrm{t} 1$ - mid-intervention, $\mathrm{t} 2$ - post-intervention, $\mathrm{t} 3$ - follow-up) with the respective measurements (primary, secondary outcome measures and key interventions including structured interviews of OASIS) and number of participants in the different steps of the study. The $\mathrm{t} 3$ - follow-up questionnaire three months after the intervention was answered by 13 participants. Due to the small sample size, follow-up data was not included in the analysis 
Table 1. Overview of the key interventions of the two webcam-based telemedicine consultation sessions

\begin{tabular}{ll}
\hline Telemedicine consultation & \multicolumn{1}{c}{ Key interventions } \\
\hline 1. Consultation session & -M.I.N.I - structured clinical Interview to verify psychiatric disorders (Sheehan et al., 1998) \\
& -structured interview to verify an IUD following the nine criteria of the DSM-5 for IGD \\
& (American Psychatric Association, 2013) \\
& -ADHS Screening (World Health Organisation, 2003) \\
& -history of Internet use \\
& -social-, family history \\
& -homework: individualized small behavior changes, first small changes in the daily behavior \\
& of Internet use for example one hour before sleeping and one hour after awaking no media \\
& use, etc.) \\
& -individual treatment recommendation near place of residence \\
& - MI techniques to increase the motivation to change one's behavior \\
& - discussing strategies to reduce Internet usage, structure everyday life \\
& -planning next small steps
\end{tabular}

(complete participation: participants who attended the entire intervention; partial participation: participants who took part in at least the first online consultation session) in order to identify possible explanations for the high dropout rates and to identify different person-related characteristics (see Table 2).

\section{Measures}

iSOCRATES. The primary outcome measure was the motivation to change the Internet use assessed by the factor "taking steps" of the iSOCRATES. Due to the fact that the intervention included a maximum of two telemedicine sessions, it was important to choose a particularly sensitive instrument, which captured a specific aspect concerning the concrete changes in one's own Internet use. The two additional factors "ambivalence" and "recognition" were additional outcome measures.

The iSOCRATES is a modified form of the SOCRATES (Miller \& Tonigan, 1997), adapted for Internet use. The selfreport measure assesses the stage of readiness and treatment eagerness of IUD. On a five-point Likert scale from "strongly disagree" to "strongly agree" the participants indicated how much they agree with the 19 statements regarding their own Internet use. The iSOCRATES consists of three factors: ambivalence (sumscore of 4 items; e.g. "Sometimes I wonder if I am in control of my Internet use."), recognition (sumscore of 7 items; e.g. "I am an Internet addict.") and taking steps (sumscore of 8 items; e.g. "I'm not just thinking about changing my Internet use, I'm already doing something about it."). Internal consistency (Cronbach's alpha) of the iSOCRATES for each factor was good/moderate: $\alpha=0.678$ (ambivalance), $\alpha=$ 0.893 (recognition), $\alpha=0.869$ (taking steps).

s-IAT \& Internet usage time. Secondary outcome measures were the reduction of the symptom severity based on the sumscore of the short Internet Addiction Test (s-IAT; Pawlikowski, Altstötter-Gleich, \& Brand, 2013), and the selfreported time spent online (hours per day (hrs/d)).

The s-IAT is a self-report measure, which assesses symptoms of IUD by 12 items on a five-point Likert scale from 1 (=never) to 5 (=very often). Sum scores were calculated ranging from 12 to 60 with scores $>30$ indicating a problematic Internet use and scores $>37$ indicating a pathological Internet use. The s-IAT can be divided in two sixitem factors: loss of control/time management and craving/ social problems. Internal consistency (Cronbach's alpha) of the $\mathrm{s}$-IAT was good/moderate: $\alpha=0.825$ (sumscore), $\alpha=$ 0.714 (loss of control/time management), $\alpha=0.804$ (social problems/craving).

IUD. The DSM-5 criteria for IGD (American Psychatric Association, 2013) were used as a basis for the self-developed guideline to diagnose an IUD in a structured way. First, the specific type of IUD was assessed by exploring which Internet content is used to a problematic extent. Second, the nine DSM-5 criteria related to the specific type of IUD were explored and assessed by the trained consultant using the structured guideline. An IUD was diagnosed if at least five of the nine DSM-5-criteria were present over a 12-month period. Hazardous Internet use was diagnosed if at least three criteria were met.

Psychiatric comorbidity. The Mini-International Neuropsychiatric Interview (M.I.N.I; Sheehan et al., 1998) is a structured diagnostic interview to identify the psychiatric disorders covered by DSM-4 and ICD-10. With an administration time of approximately 15-20 min, it was designed to meet the need of a short structured interview which covers the different psychiatric disorders (affective disorders, anxiety disorders, post-traumatic stress disorder, substance dependence, psychotic disorders, and eating disorders). 
Table 2. Comparison of participants who attended the entire study including the completion of the post intervention questionnaire (complete participation) and participants who took part in at least the first online consultation session but did not complete all questionnaires/participate at all telemedicine consultations (partial participation) with regard to sociodemographics, Internet use, comorbidities, symptoms severity of IUD and motivation to change something about their own Internet use behavior

\begin{tabular}{|c|c|c|c|c|c|c|c|}
\hline & \multirow{2}{*}{\multicolumn{3}{|c|}{$\begin{array}{c}\text { Complete participation } \\
n=73 \\
\end{array}$}} & \multirow{2}{*}{\multicolumn{3}{|c|}{$\begin{array}{c}\text { Partial participation } \\
\qquad n=67\end{array}$}} & \multirow[b]{3}{*}{ Statistics } \\
\hline & & & & & & & \\
\hline & M & SD & $\min -\max$ & M & SD & $\min -\max$ & \\
\hline Age & 34.92 & 12.49 & $19-73$ & 31.39 & 14.07 & $18-70$ & $\begin{aligned} t(138)= & 1.573, p=0.118,95 \%-C I \\
& {[-0.908,7.968] }\end{aligned}$ \\
\hline Internet use on weekdays (hrs/d) & 6.38 & 3.79 & $0.5-18$ & 6.25 & 3.57 & $0.5-15$ & $\begin{aligned} t(138)= & 0.203, p=0.840,95 \%-C I \\
& {[-1.107,1.359] }\end{aligned}$ \\
\hline Internet use on weekends (hrs/d) & 7.02 & 4.35 & $0.5-18$ & 7.07 & 3.91 & $0.5-17$ & $\begin{array}{c}t(138)=-0.072, p=0.943,95 \%-C I \\
{[-1.104,1.356]}\end{array}$ \\
\hline Male & \multicolumn{3}{|c|}{$83.6 \%(n=61)$} & \multicolumn{3}{|c|}{$86.6 \%(n=58)$} & $X^{2}(1)=0.248, p=0.619, V=0.042$ \\
\hline $\begin{array}{l}\text { One or more comorbidities diagnosed } \\
\left.\text { (M.I.N.I. }{ }^{\mathrm{a}}\right)\end{array}$ & \multicolumn{3}{|c|}{$49.3 \%(n=36)$} & \multicolumn{3}{|c|}{$30.2 \%(n=19)$} & $X^{2}(2)=8.947, p=0.011, V=0.253$ \\
\hline $\begin{array}{l}\text { Diagnosed comorbidites (including } \\
\text { multiple diagnoses) }\end{array}$ & \multicolumn{3}{|c|}{$n=47$} & \multicolumn{3}{|c|}{$n=24$} & \\
\hline Affective disorders & \multicolumn{3}{|c|}{$57.4 \%(n=27)$} & \multicolumn{3}{|c|}{$62.5 \%(n=15)$} & $X^{2}(1)=0.107, p=0.743, V=0.044$ \\
\hline Suspicious of $\mathrm{ADHD}^{\mathrm{b}}$ & \multicolumn{3}{|c|}{$31.9 \%(n=15)$} & \multicolumn{3}{|c|}{$20.8 \%(n=5)$} & $X^{2}(1)=1.266, p=0.260, V=0.152$ \\
\hline Anxiety disorder & \multicolumn{3}{|c|}{$8.5 \%(n=4)$} & \multicolumn{3}{|c|}{$16.7 \%(n=4)$} & $X^{2}(1)=0.711, p=0.399, V=0.114$ \\
\hline Eating disorder & \multicolumn{3}{|c|}{$2.2 \%(n=1)$} & \multicolumn{3}{|c|}{$0 \%$} & $X^{2}(1)=0.538, p=0.463, V=0.099$ \\
\hline \multicolumn{8}{|l|}{ Symptom severity $\left(\mathrm{s}-\mathrm{IAT}^{\mathrm{c}}\right)$} \\
\hline Sumscore & 41.30 & 8.01 & $22-58$ & 40.45 & 8.08 & $21-55$ & $\begin{aligned} t(138)= & 0.627, p=0.532,95 \%-C I \\
& {[-1.838,3.545] }\end{aligned}$ \\
\hline Loss of control \& time management & 22.71 & 4.17 & $12-30$ & 22.36 & 4.40 & $11-30$ & $\begin{aligned} t(138)= & 0.489, p=0.625,95 \%-C I \\
& {[-1.077,1.785] }\end{aligned}$ \\
\hline Craving \& social problems & 18.59 & 5.10 & $10-29$ & 18.10 & 4.47 & $7-26$ & $\begin{aligned} t(138)= & 0.614, p=0.540,95 \%-C I \\
& {[-1.109,2.109] }\end{aligned}$ \\
\hline \multicolumn{8}{|l|}{ Motivation to change (iSOCRATES ${ }^{\mathrm{d}}$ ) } \\
\hline Taking steps & 24.19 & 7.01 & $8-39$ & 23.53 & 6.13 & $9-37$ & $\begin{aligned} t(138)= & 0.599, p=0.550,95 \%-C I \\
& {[-1.539,2.878] }\end{aligned}$ \\
\hline Ambivalence & 15.19 & 3.30 & $7-20$ & 15.00 & 2.64 & $8-19$ & $\begin{aligned} t(138)= & 0.407, p=0.685,95 \%-C I \\
& {[-0.798,1.211] }\end{aligned}$ \\
\hline Recognition & 28.41 & 5.53 & $9-35$ & 27.30 & 6.01 & $10-35$ & $\begin{aligned} t(138)= & 0.1 .141, p=0.256,95 \%-C I \\
& {[-0.815,3.040] }\end{aligned}$ \\
\hline
\end{tabular}

Abbreviations: ${ }^{\mathrm{a}}$ Mini-International Neuropsychiatric Interview; complete participation: $n=2$ no M.I.N.I conducted, partial participation: $n=8$ no M.I.N.I conducted; ${ }^{\mathrm{b}}$ attention deficit/hyperactivity disorder; ${ }^{\mathrm{c}}$ short Internet Addiction Test; ${ }^{\mathrm{d}}$ stage of readiness and treatment eagerness of IUD.

ADHD screening. The screening test V1.1 is a self-assessment scale for adults to determine if a suspicion of attention deficit hyperactivity disorder (ADHD) might exist (World Health Organisation, 2003). Six questions were answered on a 5-point Likert scale from 1 "never" to 5 "very often". If four of the six responses are in the conspicuous range, it indicates a possible adult ADHD. The conspicuous range is defined as follows: Questions 1-3: at least "sometimes" (3) selected; Questions 4-6: at least "often” (4) selected.

The structured interviews of IUD and comorbidities were conducted in the first telemedicine consultation session.

\section{Statistical analysis}

Descriptive statistical analyses were calculated using percentages for categorical variables and means, standard deviation, range of scores and 95\% confidence interval for continuous variables. Independent-sample t-tests (2-sided) were conducted to compare the group of "complete participation" with "partial participation". Chi-squared tests were used for nominal variables.

Primary and secondary outcome measures were analyses of variance for within-participant comparisons over time with bonferroni-adjusted post hoc tests. Eta-square was used as effect size indicator with $\eta^{2}>0.003$ no effect, $\eta^{2}>0.039$ small, $\eta^{2}>0.110$ moderate and $\eta^{2}>0.140$ large effect. Cramer's V was calculated for the Chi-squared test with $V=0.1$ indicated small, $V=0.3$ medium and $V=0.5$ large effect (Cohen, 2013).

All analyses were conducted with IBM SPSS statistics for windows, version 26.0 and Microsoft Excel, version 16.0.

\section{Ethics}

The study was carried out in accordance with the Declaration of Helsinki. The Institutional Review Board approved the study (Nr. 16-5734). All subjects were informed about the study and all provided informed consent. 


\section{RESULTS}

\section{Study participants}

As shown in Fig. 1, a total of 284 persons provided informed consent. 144 of the 284 persons did not continue to participate in the study, 86 of these people only created a user account and 58 people completed only the questionnaires, but did not book an appointment. 140 participants completed the baseline measurement $(\mathrm{t} 0)$ and attended the first telemedicine consultation session. T1 (mid-intervention) was completed by 125 participants, 107 participants participated in the second telemedicine consultation session and 73 participants answered the questionnaires after the two telemedicine consultations ( $\mathrm{t} 2$; post-intervention). The follow-up questionnaire $\mathrm{t} 3$ three month after the second telemedicine consultation was answered by 13 participants. Due to the small sample size, follow-up data was not included in the analysis. A post-hoc power analysis was performed for the repeated measures analyses of variance with G*Power V3.1.9.7 (Faul, Erdfelder, Buchner, \& Lang, 2009). With the effect size $f=0.629$ of the primary outcome measure "taking steps" of the iSOCRATES with $n=73$ and the alpha error probability ( $\alpha$ err prob) $=0.05$, a Power $(1-\beta$ err prob) $=0.99$ was calculated.

To identify possible explanations for the high dropout rates and to ensure that there were no person-related differences between those who participated in the entire intervention including the post intervention questionnaire (complete participation) and those who did not participate in the entire intervention (partial participation), these two groups were compared with regard to several person-related factors (see Table 2). Participants in the complete participation group were diagnosed with at least one comorbidity significantly more often than those in the partial participation group. There were no significant differences between the two groups with regard to Internet usage times, symptom severity or motivation to change.

Of the 73 participants who completed the post intervention questionnaire, $83.6 \%(n=61)$ were male. Mean (SD) age was 34.92 (12.49) years (range: 19-73 years). The majority of participants $(78.1 \%, n=57)$ have a high level of education ( $\geq 12$ years of education), $16.4 \%$ a moderate $(n=$ 12 ; 11 years of education), $2.7 \%$ low level of education ( $n=$ 2 ; $>9$ years of education) and $2.7 \%(n=2)$ are currently students. $49.3 \%(n=36)$ were employed, $26 \%(n=19)$ are currently studying and $16.4 \%(n=12)$ are unemployed. $46.6 \%(n=34)$ stated that they are single, $36.2 \%(n=25)$ in a relationship, $12.3 \%(n=9)$ married, $4.1 \%(n=3)$ divorced and $2.7 \%(n=2)$ widowed.

In the course of the structured interview of IUD 58.9\% ( $n$ $=43$ ) of the participants were diagnosed with IUD, 34.2\% ( $n$ $=25)$ with hazardous Internet use and $6.8 \%(n=5)$ with subjectively burdening Internet use. Within this sample $(n=$ 73 ), online computer games were used most frequently to a problematic extent $(30.1 \% ; n=22)$, followed by Internet pornography $(26 \% ; n=19)$, information research $(17.8 \% ; n$ $=13)$, social network sites $(9.6 \% ; n=7)$, generalized

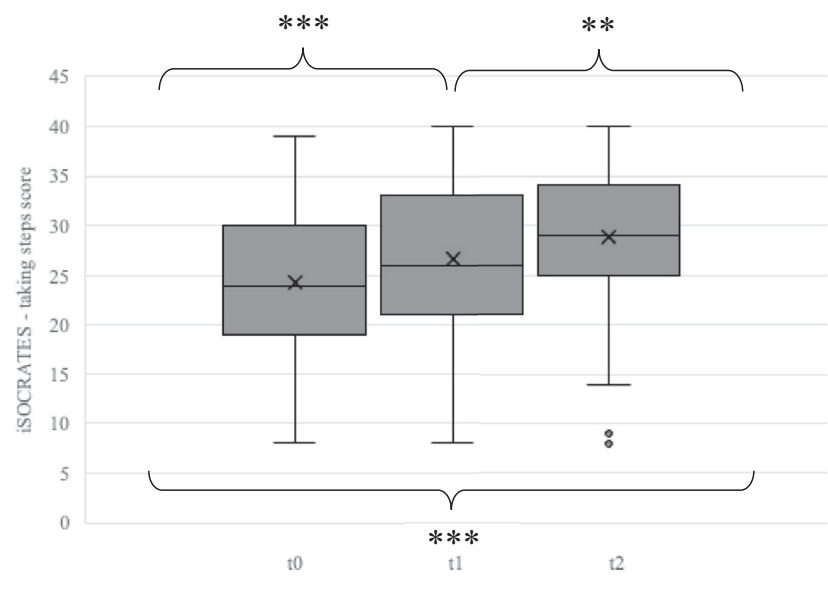

Fig. 2. Changes of the mean subjective assessment of taking steps to change the Internet use $(n=71)$.

Comparison of taking steps to change the Internet use over the three times of measurement.

The horizontal line in the middle of each box indicates the median. The cross within each box indicates the mean. Top and bottom borders of the box mark the 75th and 25th percentiles, respectively.

The whiskers above below the box mark the 90th and 10th

percentiles. The circles beyond the whiskers are outliers. Note: ${ }^{* *} p<0.01 ;{ }^{* * *} p<0.001$

Internet use $(8.3 \% ; n=6)$, streaming $(6.8 \% ; n=5)$ and online shopping $(1.4 \% ; n=1)$.

\section{Analysis of primary outcome measure}

Based on the information provided by the participants regarding the "taking steps" factor of the iSOCRATES after the last consultation, the participants took significantly more active steps to change their own Internet use behavior (average increase of 4 points) than before the intervention.

Figure 2 shows the iSOCRATES score for the factor "taking steps" over the three times of measurement ( $\mathrm{t} 0$ pre-intervention, $\mathrm{t} 1$ mid-intervention, $\mathrm{t} 2$ post-intervention). A repeated measures ANOVA with a Greenhouse-Geisser correction determined that mean score of taking steps showed a statistically significant difference between the three measurements, $F(1.816)=27.768, p<0.001, \eta^{2}=0.284$. Bonferroni-adjusted post-hoc analysis revealed a significant difference $(p<0.001)$ between the three times of measurement regarding taking steps to change the Internet use behavior (see Table 3).

\section{Analysis of secondary outcome measures}

Analysis of variance revealed significant effects over the three times of measurement for the symptom severity (s-IAT sumscore) $F(1.693)=15.958, p<0.001, \eta^{2}=0.181$ and for the two factors craving/social problems $F(2)=8.301$, $p<0.001, \eta^{2}=0.103$ and loss of control/time management, $F(1.614)=17.726, p<0.001, \eta^{2}=0.198$ (see Table 3 and Fig. 3). The post hoc analyses (see Table 3 ) showed a significant reduction in the symptom severity of IUD (sumscore and both factors) from t1 to $t 2$. No significant reduction in symptom severity (sumscore and both factors; 

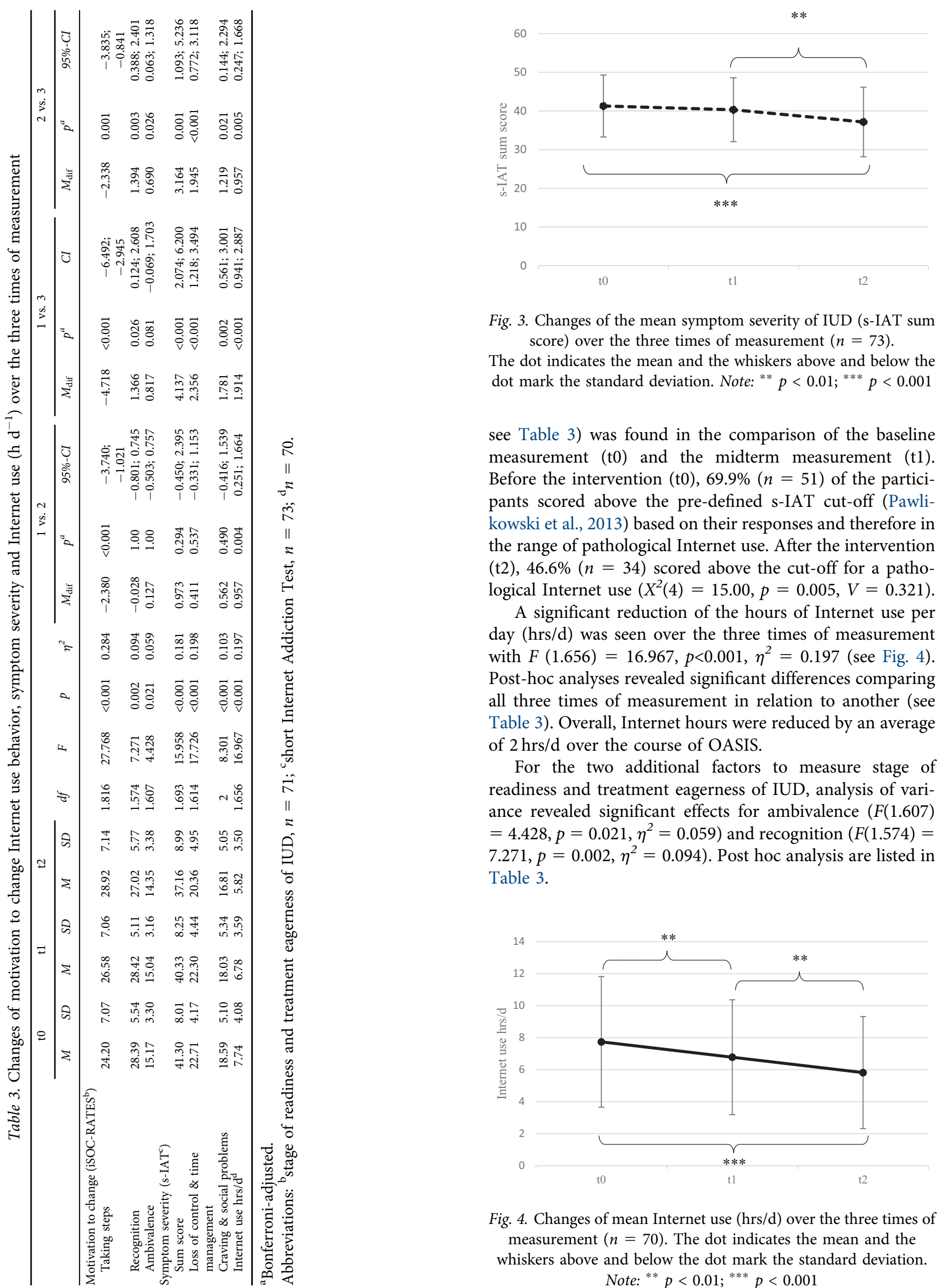

Fig. 3. Changes of the mean symptom severity of IUD (s-IAT sum score) over the three times of measurement $(n=73)$.

The dot indicates the mean and the whiskers above and below the dot mark the standard deviation. Note: ${ }^{* *} p<0.01$; ${ }^{* * *} p<0.001$

see Table 3) was found in the comparison of the baseline measurement ( $\mathrm{t} 0$ ) and the midterm measurement ( $\mathrm{t} 1)$. Before the intervention ( $\mathrm{t} 0), 69.9 \%(n=51)$ of the participants scored above the pre-defined s-IAT cut-off (Pawlikowski et al., 2013) based on their responses and therefore in the range of pathological Internet use. After the intervention $(\mathrm{t} 2), 46.6 \%(n=34)$ scored above the cut-off for a pathological Internet use $\left(X^{2}(4)=15.00, p=0.005, V=0.321\right)$.

A significant reduction of the hours of Internet use per day (hrs/d) was seen over the three times of measurement with $F(1.656)=16.967, p<0.001, \eta^{2}=0.197$ (see Fig. 4). Post-hoc analyses revealed significant differences comparing all three times of measurement in relation to another (see Table 3). Overall, Internet hours were reduced by an average of $2 \mathrm{hrs} / \mathrm{d}$ over the course of OASIS.

For the two additional factors to measure stage of readiness and treatment eagerness of IUD, analysis of variance revealed significant effects for ambivalence $(F(1.607)$ $\left.=4.428, p=0.021, \eta^{2}=0.059\right)$ and recognition $(F(1.574)=$ $\left.7.271, p=0.002, \eta^{2}=0.094\right)$. Post hoc analysis are listed in Table 3.

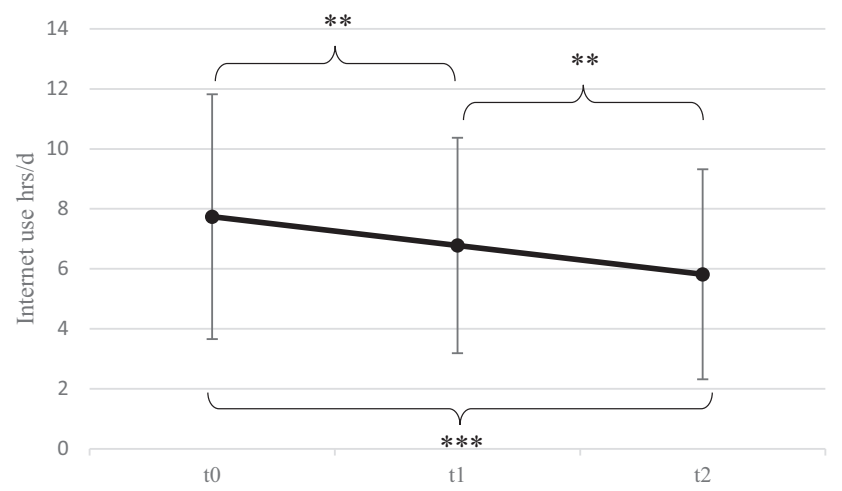

Fig. 4. Changes of mean Internet use (hrs/d) over the three times of measurement $(n=70)$. The dot indicates the mean and the whiskers above and below the dot mark the standard deviation. Note: ${ }^{* *} p<0.01{ }^{* * *} p<0.001$ 


\section{DISCUSSION}

To pick up individuals with IUD where their problem started, OASIS - a low threshold online-based telemedicine program has been developed. So far, there are very few scientific publications regarding telemedicine interventions for individuals with IUD. Already published interventions are apps or programs in which the affected person works through predefined modules independently from a professional counsellor and with almost no direct personal interaction (Chebli et al., 2016; Jonas et al., 2020; Lam \& Lam, 2016). To the best of our knowledge, these are the first published results on the efficacy of a brief telemedicine intervention for individuals with IUD involving direct webcam-based contact between the participant and counsellor.

The most important finding of this study is that already two telemedicine sessions between the participant and counsellor lead to an increase of motivation to change and first small actions of the participants to modify their Internet use behavior. The effectiveness of MI techniques via webcam for individuals with IUD could be shown, both in terms of increasing the motivation and action of the participant to change their Internet use behaviors and in terms of decreasing symptom severity and Internet use times. Onethird of the participants who were classified as having a pathological Internet use based on their responses in the symptom severity questionnaire were below the appropriate cut-off for pathological Internet use after OASIS participation. Furthermore, a reduction of Internet usage times of almost two hrs/d over the course of the OASIS program has been achieved. Thus, with an increase in motivation to change by an average of four points and an overall reduction in symptom severity of four points on mean, the pilot study demonstrated the effectiveness of the OASIS program.

The OASIS study and already published telemedicine interventions showed both similarities and differences. The majority of published interventions as well as OASIS used already established techniques as methodological basis for the intervention (Chebli et al., 2016; Jonas et al., 2020; Lam \& Lam, 2016). Already published telemedicine programs are characterized by interventions with mostly asynchronous communication in which affected individuals independently complete pre-designed modules with the primary goal of reducing the time of use and/or symptom severity of IUD. Personal contact with a counsellor is typically absent or very limited (Chebli et al., 2016; Jonas et al., 2020; Lam \& Lam, 2016). Since numerous studies on the effectiveness of psychotherapy showed that regardless of the underlying discipline, the therapeutic alliance between patient and therapist has a strong influence on in the effectiveness of therapy (Lopez, Schwenk, Schneck, Griffin, \& Mishkind, 2019; Stubbe, 2018), the therapeutic alliance was integrated as an important aspect in the concept of OASIS. A study published recently with a comparison of two telemedicine interventions for problematic gambling was able to show that increased interaction between client and counsellor led to higher efficacy (Jonas et al., 2020). Besides the way of therapeutic communication, previously published interventions and OASIS also vary in the duration of the intervention and the respective group of individuals affected. One telemedicine intervention is a one-time 30 -min Internet-based intervention based on MI techniques to treat online addiction among college students (primarily female) in China showing a strong positive effect of the intervention $(\mathrm{Su}$, Fang, Miller, \& Wang, 2011). Two telemedicine interventions for gamblers were defined by an 8-week Internet-based CBT intervention with weekly exercises (primarily male) in Finland (Castrén et al., 2013) and a 50 days web-based intervention with weekly one-to-one chat sessions for gamblers in Germany (primarily male, Jonas et al., 2020) both with strong effect sizes. Comparable effect sizes are seen for OASIS with two telemedicine webcam-based contacts and personalized consultation including individual treatment recommendation near place of residence for individuals with IUD (primarily male). Based on the different groups of study participants, type of behavioral addiction as well as duration and structure of the intervention a direct comparison of the effects of the published and present intervention is difficult. However, the results show that telemedicine interventions in their diversity are accepted by those affected and evaluated as effective.

\section{Limitations}

Several limitations should be addressed within the telemedicine OASIS pilot study. In order to strengthen the validity of the presented results, a controlled randomized study design with a follow-up measurement would be necessary. Future studies should use an already validated and established instrument for diagnosing an IUD. The outcome measures of motivational change, symptom severity, and Internet use time were collected exclusively through self-report questionnaires. An additional independent assessment of the relevant parameters by structured interviews to the different times of measurements would strengthen the quality and validity of the results. The results show that primarily affected males participated in the telemedicine study, which is why an implication for female affected persons is only possible to a certain extent. Epidemiological studies reveal no statistically significant gender differences in the prevalence of IUD (Rumpf et al., 2014), which justifies a more balanced inclusion of men and women with IUD in future studies. Over the course of the OASIS pilot study, high dropout rates were found. In order to identify possible reasons for the high dropout rates, the participants who took part in the entire intervention (complete participation) were compared with the participants who took part in at least the first telemedicine consultation (partial participation) with regard to personal characteristics. It appeared that in the group of complete participation significantly more complex affected persons (at least one comorbidity) were identified than in the group of partial participation. It shows that telemedicine services are used by both less complex as well as complexly affected individuals. Still the whole offer of the project, including the relay into health care structures, is primary received by those suffering from complex psychopathology. The dropout rates 
are consistent with the findings of Brorson, Arnevik, RandHendriksen, and Duckert (2013), who reported dropout rates of $23-50 \%$ in a systematic review among young adults who were in addiction treatment. The collection of followup data proved to be particularly difficult, meaning that no reliable conclusions could be made regarding long-term effects of the telemedicine intervention due to the low response rate. Future studies should place a clear focus on reduce drop-outs and especially collecting follow-up data while developing strategies to strengthen the commitment of the participants through brief contacts via webcam at the questionnaire measurement points, compensation of expenses and/or possibility to remind participants to complete the questionnaires both by e-mail and by telephone.

\section{CONCLUSION}

The findings of the present study supplement the currently very limited state of existing telemedicine interventions for individuals with IUD. To the best of our knowledge, these are the first published results on the efficacy of a brief telemedicine intervention for individuals with IUD involving two webcam-based contacts between the participant and counsellor. Since the acceptance and dissemination of telemedicine services is increasing, the promising results of the present study are of high relevance for research and practice. Should the effectiveness of telemedicine services be confirmed in randomized controlled trails with follow-up measurements, it would be a promising approach to extent the already existing health care system with low-threshold, location-independent specialized telemedicine services as preventive measure to avoid the development of addiction symptoms and chronification of IUD.

Funding: This publication was funded by the German Federal Ministry of Health under grant number ZMVI12516 DSM207.

Authors' contribution: LB wrote the manuscript. LB, JDH, BTW created the manuscript concept. BTW conceived the study and acquired funding. LB and BTW conducted and coordinated the study. $\mathrm{MB}, \mathrm{SH}, \mathrm{NT}$ contributed to the study design and refinements and approved the final version of the protocol. MB and BTW supervised manuscript writing. All authors approved the final version of the manuscript.

Conflict of interest: The authors declare no conflict of interest.

Acknowledgements: We acknowledge support by the Open Access Publication Funds of the Ruhr-University Bochum. Our thanks go to the German Federal Ministry of Health for sponsoring the study and to the cooperation partners Centre for Telematics and Telemedicine (ZTG) GmbH and the German Fachverband Medienabhängigkeit e.V.

\section{REFERENCES}

American Psychatric Association. (2013). Diagnostic and statistical manual of mental disorders: DSM-5. Washington, DC: American psychiatric association.

Bischof, G., Bischof, A., \& Rumpf, H. J. (2021). Motivational interviewing: An evidence-based approach for use in medical practice. Deutsches Arzteblatt International, 7(118), 109-115. https://doi.org/10.3238/arztebl.m2021.0014.

Bommelé, J., Schoenmakers, T. M., Kleinjan, M., Peters, G.-J. Y., Dijkstra, A., \& van de Mheen, D. (2017). Targeting hardcore smokers: The effects of an online tailored intervention, based on motivational interviewing techniques. British Journal of Health Psychology, 22(3), 644-660. https://doi.org/10.1111/bjhp.12256.

Brand, M., Rumpf, H.-J. ., Demetrovics, Z., Mueller, A., Stark, R., King, D. L., .. Potenza, M. N. (2020). Which conditions should be considered as disorders in the International Classification of Diseases (ICD-11) designation of "other specified disorders due to addictive behaviors"? Journal of Behavioral Addictions. https://doi.org/10.1556/2006.2020.00035.

Brand, M., Young, K. S., Laier, C., Wölfling, K., \& Potenza, M. N. (2016). Integrating psychological and neurobiological considerations regarding the development and maintenance of specific Internet-use disorders: An Interaction of Person-Affect-CognitionExecution (I-PACE) model. Neuroscience and Biobehavioral Reviews, 71, 252-266. https://doi.org/10.1016/j.neubiorev.2016.08.033.

Brorson, H. H., Arnevik, E. A., Rand-Hendriksen, K., \& Duckert, F. (2013). Drop-out from addiction treatment: A systematic review of risk factors. Clinical Psychology Review, 33(8), 10101024. https://doi.org/10.1016/j.cpr.2013.07.007.

Carli, V., Durkee, T., Wasserman, D., Hadlaczky, G., Despalins, R., Kramarz, E., ... , Brunner, R.. (2013). The association between pathological internet use and comorbid psychopathology: A systematic review. Psychopathology, 46(1), 1-13. https://doi.org/ 10.1159/000337971.

Castrén, S., Pankakoski, M., Tamminen, M., Lipsanen, J., Ladouceur, R., \& Lahti, T. (2013). Internet-based CBT intervention for gamblers in Finland: Experiences from the field. Scandinavian Journal of Psychology, 54(3), 230-235. https://doi.org/10. 1111/sjop.12034.

Chebli, J. L., Blaszczynski, A., \& Gainsbury, S. M. (2016). Internetbased interventions for addictive behaviours: A systematic review. Journal of Gambling Studies, 32(4), 1279-1304. https:// doi.org/10.1007/s10899-016-9599-5.

Cohen, J. (2013). Statistical power analysis for the behavioral sciences. Academic Press.

Diclemente, C. C., Corno, C. M., Graydon, M. M., Wiprovnick, A. E., \& Knoblach, D. J. (2017). Motivational interviewing, enhancement, and brief interventions over the last decade: A review of reviews of efficacy and effectiveness. Psychology of Addictive Behaviors, 31(8). https://doi.org/10.1037/adb0000318.

Faul, F., Erdfelder, E., Buchner, A., \& Lang, A.-G. (2009). Statistical power analyses using $G^{*}$ Power 3.1: Tests for correlation and regression analyses. Behavior Research Methods, 41(4), 11491160. https://doi.org/10.3758/BRM.41.4.1149.

Hill, D. J., \& Laredo, E. (2019). First and last and always: Streetwork as a methodology for radical community social work practice. 
Critical and Radical Social Work, 7(1), 25-39. https://doi.org/ 10.1332/204986019X15491042559682.

Jiang, S., Wu, L., \& Gao, X. (2017). Beyond face-to-face individual counseling: A systematic review on alternative modes of motivational interviewing in substance abuse treatment and prevention. Addictive Behaviors, 73, 216-235. https://doi.org/ 10.1016/j.addbeh.2017.05.023.

Johansson, R., \& Andersson, G. (2012). Internet-based psychological treatments for depression. Expert Review of Neurotherapeutics, 12(7), 861-870. https://doi.org/10.1586/ern.12.63.

Jonas, B., Leuschner, F., Eiling, A., Schoelen, C., Soellner, R., \& Tossmann, P. (2020). Web-based intervention and emailcounseling for problem gamblers: Results of a randomized controlled trial. Journal of Gambling Studies, 36(4), 1341-1358. https://doi.org/10.1007/s10899-019-09883-8.

Kruse, C. S., Lee, K., Watson, J. B., Lobo, L. G., Stoppelmoor, A. G., \& Oyibo, S. E. (2020). Measures of effectiveness, efficiency, and quality of telemedicine in the management of alcohol abuse, addiction, and rehabilitation: Systematic review. Journal of Medical Internet Research, 22(1), e13252. https://doi.org/10.2196/13252.

Lam, L. T., \& Lam, M. K. (2016). eHealth intervention for problematic internet use (PIU). Current Psychiatry Reports, 18(12). https://doi.org/10.1007/s11920-016-0747-5.

Lopez, A., Schwenk, S., Schneck, C. D., Griffin, R. J., \& Mishkind, M. C. (2019). Technology-based mental health treatment and the impact on the therapeutic alliance. Current Psychiatry Reports, 21(8), 1-7. https://doi.org/10.1007/s11920-019-1055-7.

Mihara, S., \& Higuchi, S. (2017). Cross-sectional and longitudinal epidemiological studies of internet gaming disorder: A systematic review of the literature. Psychiatry and Clinical Neurosciences, 71(7), 425-444. https://doi.org/10.1111/pcn.12532.

Miller, W. R., \& Tonigan, J. S. (1997). Assessing drinkers' motivation for change: The stages of change readiness and treatment eagerness scale (SOCRATES). American Psychological Association. https://doi.org/10.1037/10248-014.

Montag, C., Bey, K., Sha, P., Li, M., Chen, Y.-F., Liu, W.-Y., ... Keiper, J. (2015). Is it meaningful to distinguish between generalized and specific internet addiction? Evidence from a cross-cultural study from Germany, Sweden, Taiwan and China. Asia-Pacific Psychiatry, 7(1), 20-26. https://doi.org/10. 1111/appy.12122.

Montag, C., Wegmann, E., Sariyska, R., Demetrovics, Z., \& Brand, M. (2021). How to overcome taxonomical problems in the study of Internet use disorders and what to do with "smartphone addiction"? Journal of Behavioral Addictions, 9(4), 908914. https://doi.org/10.1556/2006.8.2019.59.

O’Connor, L. E., Berry, J. W., Inaba, D., Weiss, J., \& Morrison, A. (1994). Shame, guilt, and depression in men and women in recovery from addiction. Journal of Substance Abuse Treatment, 11(6), 503-510. https://doi.org/10.1016/0740-5472(94)90001-9.

Pawlikowski, M., Altstötter-Gleich, C., \& Brand, M. (2013). Validation and psychometric properties of a short version of Young's Internet Addiction Test. Computers in Human Behavior, 29(3), 1212-1223. https://doi.org/10.1016/j.chb.2012.10.014.
Potenza, M. N., Higuchi, S., \& Brand, M. (2018). Call for research into a wider range of behavioural addictions. Nature, 555(7694). https://doi.org/10.1038/d41586-018-02568-z.

Rumpf, H.-J., Vermulst, A. A., Bischof, A., Kastirke, N., Gürtler, D., Bischof, G., ... Meyer, C. (2014). Occurence of internet addiction in a general population sample: A latent class analysis. European Addiction Research, 20(4), 159-166. https://doi. org/10.1159/000354321.

Saunders, J. B., Hao, W., Long, J., King, D. L., Mann, K., FauthBühler, M., ... Poznyak, V. (2017). Gaming disorder: Its delineation as an important condition for diagnosis, management, and prevention. Journal of Behavioral Addictions, 6(3), 271-279. https://doi.org/10.1556/2006.6.2017.039.

Sheehan, D. V., Lecrubier, Y., Sheehan, K. H., Amorim, P., Janavs, J., Weiller, E., ... Dunbar, G. C. (1998). The mini-international neuropsychiatric interview (MINI): The development and validation of a structured diagnostic psychiatric interview for DSMIV and ICD-10. Journal of Clinical Psychiatry, 59(20), 22-33.

Stevens, M. W. R., King, D. L., Dorstyn, D., \& Delfabbro, P. H. (2019). Cognitive-behavioral therapy for internet gaming disorder: A systematic review and meta-analysis. Clinical Psychology \& Psychotherapy, 26(2), 191-203. https://doi.org/10.1002/cpp.2341.

Stubbe, D. E. (2018). The therapeutic alliance: The fundamental element of psychotherapy. Focus, 16(4), 402-403. https://doi. org/10.1176/appi.focus.20180022.

Su, W., Fang, X., Miller, J. K., \& Wang, Y. (2011). Internet-based intervention for the treatment of online addiction for college students in China: A pilot study of the healthy online self-helping center. Cyberpsychology, Behavior, and Social Networking, 14(9), 497-503. https://doi.org/10.1089/cyber.2010.0167.

Szeintuch, S. (2015). Street work and outreach: A social work method? The British Journal of Social Work, 45(6), 1923-1934. https://doi.org/10.1093/bjsw/bcu103.

Tait, R. J., Spijkerman, R., \& Riper, H. (2013). Internet and computer based interventions for cannabis use: A meta-analysis. Drug and Alcohol Dependence, 133(2), 295-304. https://doi.org/ 10.1016/j.drugalcdep.2013.05.012.

van Rooij, A. J., Zinn, M. F., Schoenmakers, T. M., \& van de Mheen, D. (2012). Treating internet addiction with cognitive-behavioral therapy: A thematic analysis of the experiences of therapists. International Journal of Mental Health and Addiction, 10(1), 69-82. https://doi.org/10.1007/s11469-010-9295-0.

Van't Hof, E., Cuijpers, P., \& Stein, D. J. (2009). Self-help and internet-guided interventions. CNS Spectrums, 14(2), 34-40. https://doi.org/10.1017/s1092852900027279.

World Health Organisation. (2003). Screening-Test mit SelbstbeurteilungsSkala V1. 1 für Erwachsene mit ADHS (ASRS-V1. 1). Genf: WHO.

World Health Organization. (2018). Website for ICD-11 beta draft (Mortality and morbidity statistics). Retrieved from https://icd. who.int/dev11/1-m/en.

Yen, J.-Y., Ko, C.-H., Yen, C.-F., Chen, S.-H., Chung, W.-L., \& Chen, C.-C. (2008). Psychiatric symptoms in adolescents with Internet addiction: Comparison with substance use. Psychiatry and Clinical Neurosciences, 62(1), 9-16. https://doi.org/10.1111/ j.1440-1819.2007.01770.x.

Open Access. This is an open-access article distributed under the terms of the Creative Commons Attribution-NonCommercial 4.0 International License (https:// creativecommons.org/licenses/by-nc/4.0/), which permits unrestricted use, distribution, and reproduction in any medium for non-commercial purposes, provided the original author and source are credited, a link to the CC License is provided, and changes - if any - are indicated. 